\title{
Sex differences in virtual navigation influenced by scale and navigation experience
}

\author{
Lace M. Padilla ${ }^{1}$ - Sarah H. Creem-Regehr ${ }^{1}$ - Jeanine K. Stefanucci ${ }^{1}$. \\ Elizabeth A. Cashdan ${ }^{2}$
}

Published online: 6 October 2016

(C) Psychonomic Society, Inc. 2016

\begin{abstract}
The Morris water maze is a spatial abilities test adapted from the animal spatial cognition literature and has been studied in the context of sex differences in humans. This is because its standard design, which manipulates proximal (close) and distal (far) cues, applies to human navigation. However, virtual Morris water mazes test navigation skills on a scale that is vastly smaller than natural human navigation. Many researchers have argued that navigating in large and small scales is fundamentally different, and small-scale navigation might not simulate natural human navigation. Other work has suggested that navigation experience could influence spatial skills. To address the question of how individual differences influence navigational abilities in differently scaled environments, we employed both a large- (146.4 $\mathrm{m}$ in diameter) and a traditional- (36.6 $\mathrm{m}$ in diameter) scaled virtual Morris water maze along with a novel measure of navigation experience (lifetime mobility). We found sex differences on the small maze in the distal cue condition only, but in both cue-conditions on the large maze. Also, individual differences in navigation experience modulated navigation performance on the virtual water maze, showing that higher mobility was related to better performance with proximal cues for only females on the small maze, but for both males and females on the large maze.
\end{abstract}

Lace M. Padilla

u0747331@psych.utah.edu

1 Department of Psychology, University of Utah, 380 S. 1530 E., Room 502, Salt Lake City, UT 84112, USA

2 Department of Anthropology, University of Utah, Salt Lake City, UT, USA
Keywords Virtual navigation $\cdot$ Sex differences $\cdot$ Navigation experience

The ability to successfully navigate in large-scale spaces is important for many daily activities, such as navigating to work or finding one's way to new locations when traveling. Successful navigation in large-scale spaces involves the ability to determine one's relative orientation to the visual cues in the environment and to form spatial memories that incorporate the cues. A growing body of literature shows sex differences in the types of visual cues used for navigation in smaller-scale spaces (e.g., Astur, Ortiz, \& Sutherland, 1998; Chai \& Jacobs, 2010). While these findings are robust, navigating in these small-scale spaces may be fundamentally different from navigating in large-scale spaces (Hegarty, Montello, Richardson, Ishikawa, \& Lovelace, 2006; Montello, 1993). Some have argued that navigation in small spaces might not simulate natural human navigation (Learmonth, Newcombe, Sheridan, \& Jones 2008). In addition, in many populations, males have larger range sizes than females (e.g., Ecuyer-Dab \& Robert, 2004; Santos, McGuckin, Nakamoto, Gray, \& Liss, 2011; Vashro, Padilla, \& Cashdan, 2016) and studies show a relationship between spatial abilities and range size (EcuyerDab \& Robert, 2004; Vashro \& Cashdan, 2015). To determine whether both scale and navigation experience influence sex differences in cue use during navigation, we examined performance in a traditional small-scale virtual Morris water maze (Morris, 1984), a widely used spatial abilities test developed for animals and adapted for humans, and a large-scale version, along with collecting a novel measure of self-reported navigation experience.

The traditional Morris water maze reveals sexually dimorphic visual cue-use for constructing a cognitive map (Tolman, 1948). The cognitive map is a spatial mental representation, 
defined as allocentric because it preserves object-to-object spatial relationships of cues that are not tied to the viewpoint of the observer. Visual cues used for navigation are generally divided into two categories, close or proximal cues, and distant or distal cues ${ }^{1}$ (O'Keefe \& Nadel, 1978). Proximal cues primarily provide positional information during locomotion because of their greater effect on parallax, the apparent displacement of cues on the retina when the viewer changes location. Distal cues are more suited to providing stable bearing information as they translate and rotate less than proximal cues during movement (Knierim \& Hamilton, 2011). The Morris water maze differentiates the ability to utilize cues by manipulating the visibility of each cue, then testing accuracy in returning to a remembered location. Three decades of research has shown that male rats can utilize both distal and proximal cues in the Morris water maze while females predominantly rely on proximal cues (D'Hooge \& De Deyn, 2001).

Virtual adaptations of the Morris water maze have examined sex differences in cue use by humans during navigation in small-scale spaces (on average around $35 \mathrm{~m}$ ). Generally the results replicate the rodent work, with males showing greater navigational ability when presented with distal cues alone, compared to females, and a smaller or no sex difference given the presence of proximal cues (e.g., Astur et al., 1998; Chai \& Jacobs, 2009; Sandstrom, Kaufman, \& Huettel, 1998). However, little is known about how distal and proximal cues are used in larger scales compared with those used by traditional virtual Morris water mazes, an important question given that larger scales more closely approximate natural human navigation (Learmonth, Newcombe, Sheridan, \& Jones, 2008). For example, the US Department of Transportation reported that average 21- to 35-year-olds travel 37.7 miles per day, with average trip length at 9.7 miles (Santos et al., 2011) and there is evidence that males travel more than females both in the USA (Santos et al., 2011) and crossculturally (e.g., Cashdan \& Gaulin, 2016; MacDonald \& Hewlett, 1999). Individual differences in egocentric and allocentric strategies are commonly used to explain the sex differences found in the Morris water maze. Generally, males more often use allocentric or orientation strategies, encoding the relative organization, directions, distances, and distributions of geographic landmarks (both proximal and distal) in a cognitive map (Livingstone-Lee et al., 2011). In contrast, females more often use egocentric or route-based strategies, organizing the relative geography in relation to their current location, and thus may rely more on proximal cues (e.g., Choi,

\footnotetext{
${ }^{1}$ There is not consistency between terminology, classification, and function of close cues (e.g, proximal (Vorhees \& Williams, 2006; Knierim \& Hamilton, 2011), positional (Chai \& Jacobs, 2010), landmark (Sandstrom, Kaufman, \& Huettel, 1998), and apparatus cues (Knierim \& Hamilton, 2011)) and far cues (e.g., distal (Vorhees \& Williams, 2006), directional, survey, beacon, and bearing cues (Chai \& Jacobs, 2010).
}

McKillop, Ward, \& L'Hirondelle, 2006; Lawton, 1994; Nadel $\&$ Hardt, 2004). In a large-scale environment where there are greater distances between proximal landmarks and the distal landmarks are overall farther away (as in our current study), there may be differences in how these cues are utilized. For example, females who rely on egocentric landmark strategies may suffer on performance when proximal cues are farther apart. Implementing a large-scale maze can thus help to expand our understanding of how and when these different cues are used.

Given our focus on a large-scale environment that more closely approximates the scale of human navigation, experience with navigation - such as how much individuals travel - becomes an important variable to consider. Some research suggests that sex differences in range size can predict spatial abilities including mental rotation and object location memory in western populations (Ecuyer-Dab \& Robert, 2004; Jones, Braithwaite, \& Healy, 2003) as well as in hunter-gatherer societies (e.g., Vashro \& Cashdan, 2015). Other related research on spatial cognitive development shows that older children, who have more practice navigating in large-scale spaces than younger children, can generalize their experience to spatial tasks in small-scale spaces while younger children show difficulty performing the same task in large and small spaces (Learmonth et al., 2002). Thus, our goals were two-fold. First, we examined whether the established sex difference found in maze performance with distal cues alone, but not proximal, would replicate in a large-scale maze. We expected to find sex differences in both small- and large-scale mazes, but also that the difference might increase or occur in both cueconditions in the large-scale maze. Second, we tested whether mobility experience, as assessed through selfreported travel, would modulate the predicted sex difference on maze performance. One possible outcome was that people would show enhanced navigational skills on a scale that more closely matches the scale of their natural navigation, predicting facilitatory effects of mobility only for the large-scale maze. Alternatively, highly mobile people might show better performance when navigating at both scales, consistent with a more broad relationship between mobility and spatial abilities (EcuyerDab \& Robert, 2004).

\section{Method}

\section{Participants}

One hundred and eight University of Utah undergraduates (54 male, 54 female, aged $18-55$ years, $M=23$ years) were tested 
with 54 participants ( 27 male, 27 female) each randomly assigned to either the large- or small-scale environments.

\section{Apparatus}

Virtual mazes were created using video game software (Unity Technologies, 2015). A Logitech Extreme 3D Pro Joystick was used to navigate in the virtual mazes that were displayed on a $1,920 \times 1,080$ pixel resolution monitor with SRGB color. The speed of movement was fixed to $1 \mathrm{~m} / \mathrm{s}$ and eye height was positioned in the center of the monitor with a viewing distance of $25 \mathrm{in}$. Participants used the joystick to move forward and turn from left to right, which is consistent with prior human and animal studies.

Virtual mazes In traditional virtual water maze tasks, participants search in a small pool in a virtual room with a hidden platform after which their ability to return to the platform with varying visual stimuli is measured. We adapted this paradigm to a natural landscape more closely related to a navigation task that could occur in the physical world. Participants navigated in a large grassy field and searched for a grouping of red hummingbirds, which were visible (see Fig. 1). As in Chai and Jacobs (2009), the small-scale maze was $36.6 \mathrm{~m}$ in diameter, the new large-scale maze was $146.4 \mathrm{~m}$ in diameter. In the proximal cue condition, the visual cues were close trees, bushes, and flowers of varying sizes and colors. In the proximal condition, of the larger scale maze, because it was an open field, the participant could likely detect the presence of all of the cues from one viewing location, but it was not possible to see their specific features or identify them without moving. The distal cue condition contained only cues past $100 \mathrm{~m}$, such as mountains, hills, sunset, and clouds (see Fig. 2). Each maze was contained by an invisible circular fence that could not be passed through.

Navigation experience We designed a navigation experience questionnaire that was intended to provide a broad measure of mobility. The participants viewed a list of locations in Utah (local) and in the USA (national) and indicated the locations that they had visited. The local locations provided a balanced distribution of locations that could be navigated to by car or public transportation, in addition to locations that were within walking or biking distance from the University of Utah. For Utah, there were 41 locations, with a mean driving distance from the University of Utah of 91.2 miles. Outside of Utah, there were 13 regions in the USA listed (see Supplementary materials for full mobility questionnaire). While there are some limitations to our lifetime mobility measure: our intention was to determine whether participants had traveled to many locations that likely required novel navigation practice, which may be more spatially demanding than distances or places traveled to on a daily basis (as typically tested in measures of home range size). A mobility score was calculated by totaling the number of 41 locations in Utah and 13 regions in the USA that the participant had visited.

\section{Design and procedure}

Scale (large or small) was varied between-participants and cue-type (proximal or distal) was varied withinparticipants (blocked and counterbalanced for order). After giving consent, participants had a brief practice period to gain experience using the joystick. At the start of the experiment, participants navigated with the joystick to locate a group of red birds in the virtual environment. Once the birds were located, participants were instructed to look around the environment (by rotating their viewpoint) and then they were virtually transported to a new starting location. On six subsequent trials, participants were asked to return to the location of the birds, which were hidden from view. On each trial, after participants indicated that they were at the location, the birds appeared, and the participants were encouraged to look around the environment to locate the birds. The procedure was then repeated with the second visualcue condition. Performance accuracy was measured by recording $\mathrm{X}$ and $\mathrm{Y}$ coordinates of end location, which was used to calculate the Euclidean distance from the actual location of the birds (Error). After completion of the water maze tasks, participants completed the lifetime mobility questionnaire and a measure of video gaming experience (rating $0-4$, never played 3D games to play 3D games more than twice per week). Participants also completed a battery of spatial abilities tasks as part of a larger project, but these were not analyzed in the context of the goals of the present study.

\section{Results}

To test the effect of maze scale and navigation experience (Mobility) on sex differences in cue use in the virtual Morris water maze, we conducted a linear mixed-effects analysis on the small-scale and large-scale maze error separately. ${ }^{2}$ In each analysis, lme4 in R (Bates, Maechler, \& Bolker, 2012) was used to calculate the regression weights and $3 \mathrm{D}$ video game experience (Gaming) was entered as a covariate. Trial sequence (Trial Number), proximal and distal block order (Block Order), Sex, Cue Type (Cue), Mobility score

\footnotetext{
${ }^{2}$ Because overall chance of error was inherently greater for the largeversus small-scale maze, we did not initially run the omnibus analysis with both scales included together. However, an additional analysis was conducted where maze error for the large-scale environment was divided by four to allow for direct comparisons between the scales. The findings of this analysis were consistent with the presented results.
} 
Fig. 1 Example of the viewer's perspective in the distal (top) and proximal (bottom) cue conditions

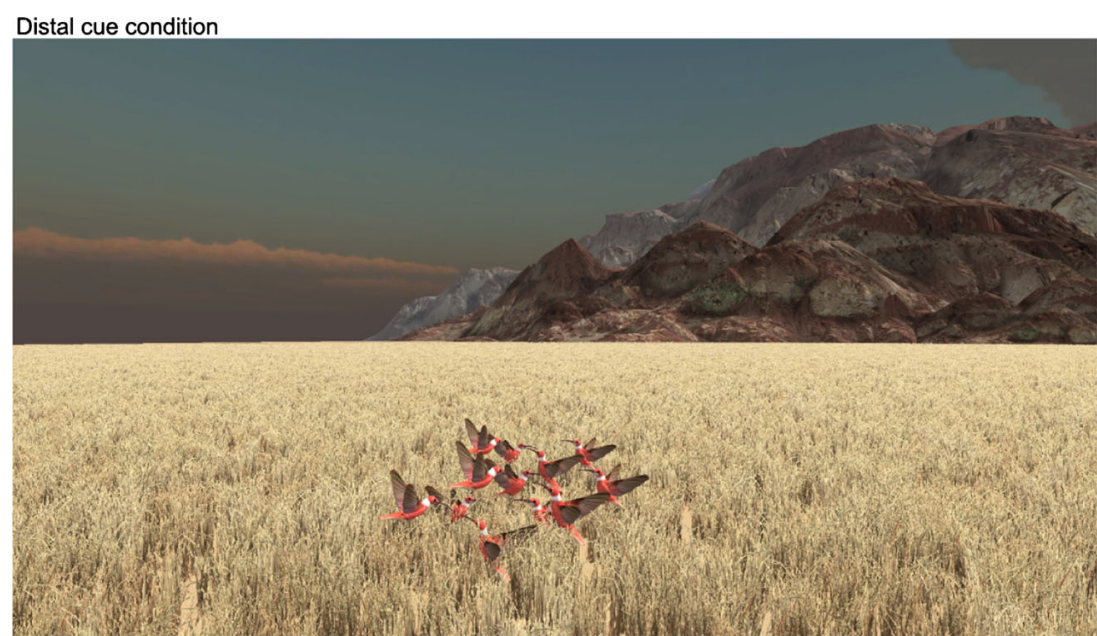

Proximal cue condition

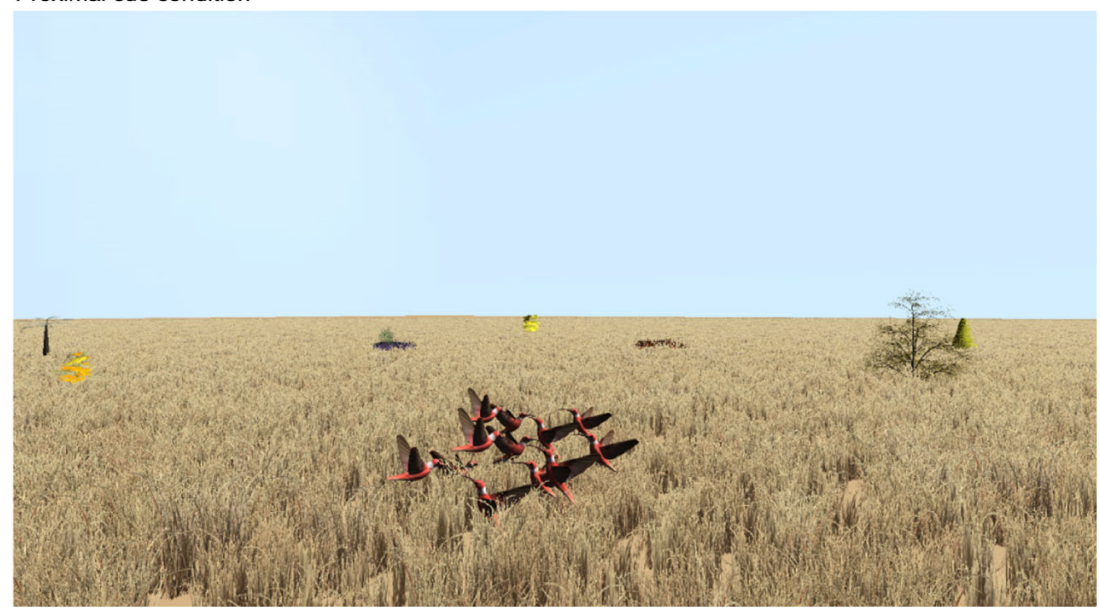

(Mobility), and the interactions between Trial Number, Block Order, Sex, Cue, and Mobility were used to predict maze performance (Error). ${ }^{3}$ A random intercept was included for each subject.

Table 1 shows a summary of the regression coefficients for the small-scale maze.

After accounting for the significant effect of Gaming our results showed a main effect of Trial Number revealing that participants' errors decreased over the sequence of trials but there was no interaction between Sex and Trial, replicating other work on practice effects (e.g., Chai \& Jacobs, 2009). There was also a significant Cue $\times$ Sex interaction (see Fig. 3) along with a Cue $\times$ Mobility interaction, which are both qualified by the significant three-way interaction between Cue, Mobility, and Sex (see Fig. 4). To understand the Cue $\times$ Sex interaction, we conducted post-hoc $t$-tests on the individual

\footnotetext{
${ }^{3}$ We used the following code in R: lmer (Error $\sim$ Game + Trial Number*Sex +Block Order*Sex + Mobility*Gender*Cue + $(1 \mid$ Subject)). $P$-values were obtained by using normal approximations.

${ }^{4}$ There was also no significant main effect of mobility for males.
}

components of the interaction. We found that when navigating with distal cues, males $(M=8.45 S E=.75)$ had significantly fewer errors than females $(M=10.80, S E=$ $.56), t(52)=2.478, p=.017,95 \%$ CI $[0.44,4.24]$. However there was no sex difference when navigating with proximal cues, $t(52)=1.65, p=.107,95 \%$ CI [$0.30,3.10]$. This relationship replicates previous findings that showed significant sex differences only for the distal cue condition (e.g., Chai \& Jacobs, 2009). To examine the relationship between Cue, Mobility and Sex, we computed separate linear regression equations for each sex and tested for the interaction between Cue and Mobility. There was a significant interaction for females, $(b=-0.10), t(320)=-2.06, p=0.039,95 \%$ CI $[-0.19,-$ $0.004]$, revealing that mobility was associated with decreased navigation errors for females in the proximal condition more than in the distal condition. However, Cue and Mobility did not interact for males $(b=0.05)$, $t(320)=1.08, p=0.28,95 \%$ CI $[-0.04,0.16]^{4}$ This suggests that navigation experience is particularly advantageous for women when proximal cues are available. It could also be the case that many females did not attend 

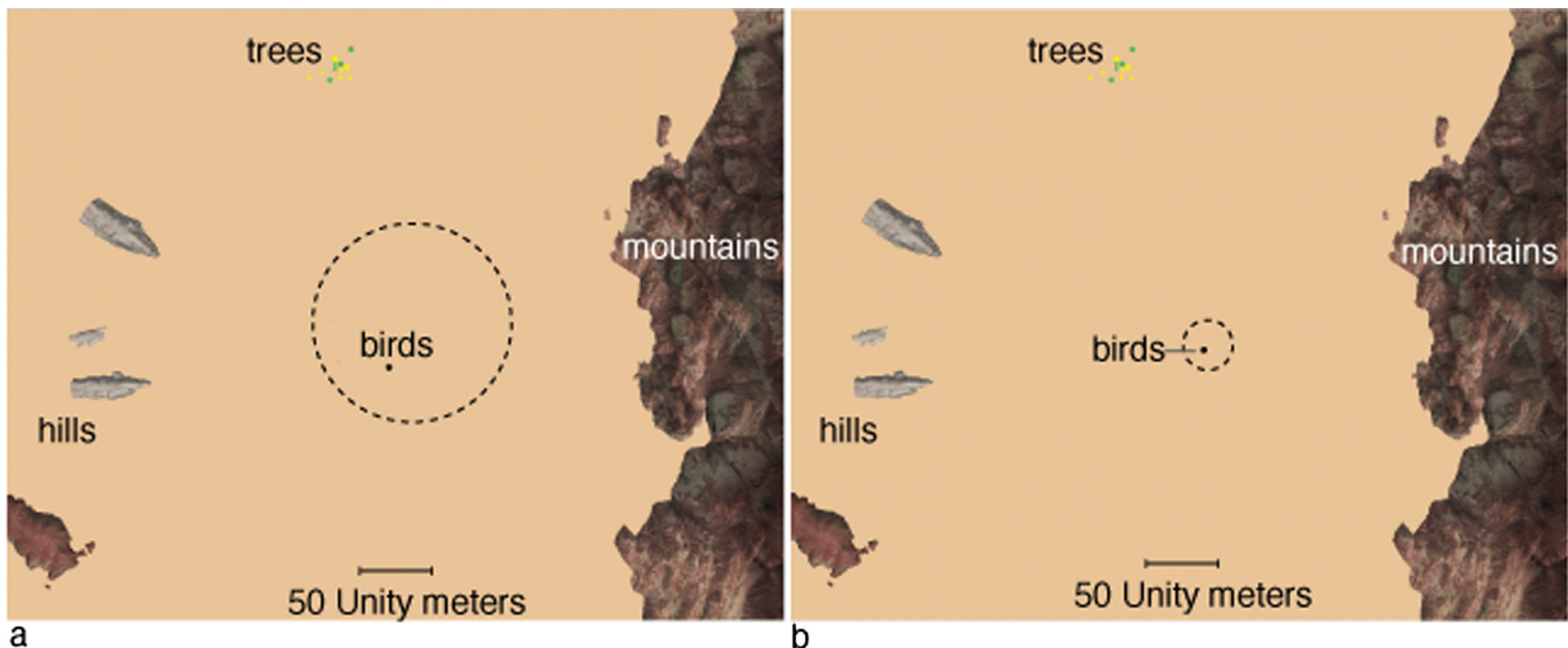

\section{T: Tree \\ F: Flowers \\ B: Bush}

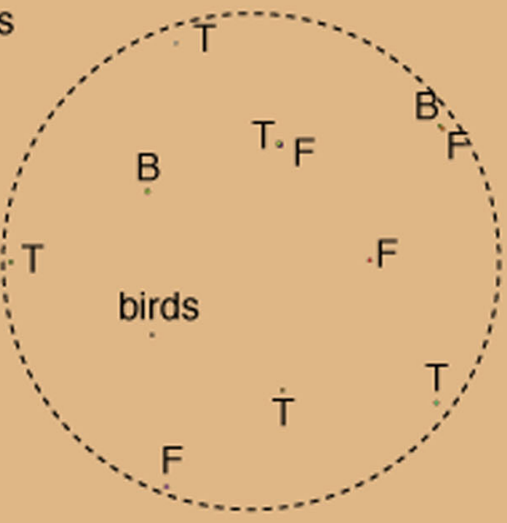

40 Unity meters b

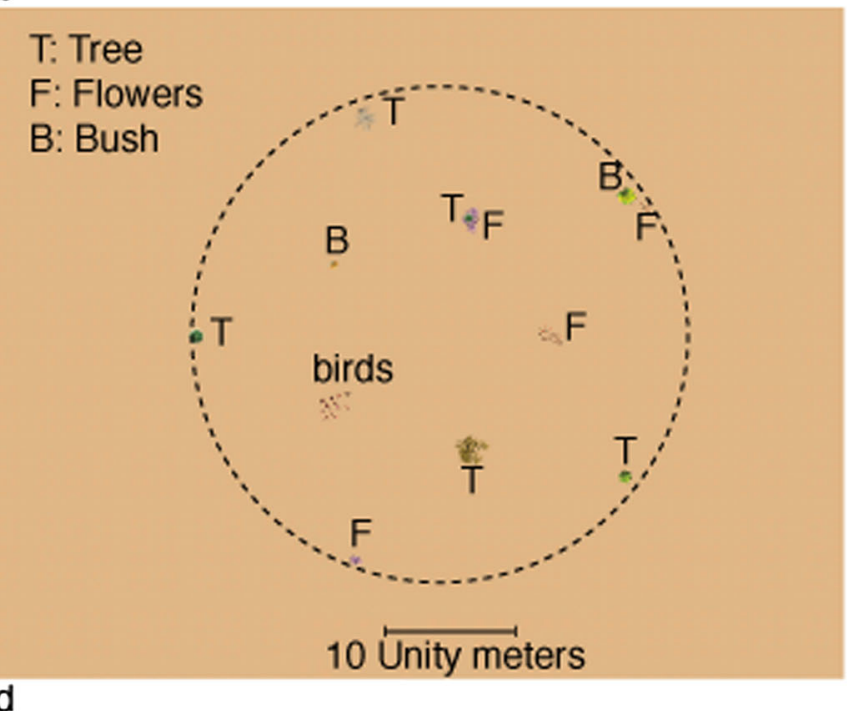

Fig. 2 Schematic diagrams, which show the visual cues and their locations from a top-down perspective in each condition. (a) Distal large scale. (b) Distal small scale. (c) Proximal large scale. (d) Proximal small scale. Participants never saw these diagrams

Table 1 List of fixed effects with coefficients, standard errors, $\mathrm{p}$ values and $95 \%$ confidence intervals from the statistical model for the small-scale maze. Coefficients for interactions including Cue indicate the change from distal to proximal cues. Coefficients for interactions including Sex indicate the change from female to male. Coefficients for interactions including Block Order indicate the change from proximal then distal to distal then proximal

\begin{tabular}{lclcll}
\hline Fixed effect & Estimate & Standard error & $t$-ratio & $p$-value & $95 \%$ CI \\
\hline Sex & -3.86 & 2.45 & -1.57 & .115 & $(-8.68,0.95)$ \\
Cue & -3.98 & 1.26 & -3.13 & $.001^{*}$ & $(-6.46,-1.49)$ \\
Mobility & -0.06 & 0.04 & -1.26 & 0.20 & $(-0.15,0.03)$ \\
Trial number & -0.28 & 0.12 & -2.31 & $0.02^{*}$ & $(-0.52,-0.04)$ \\
Gaming & -0.75 & 0.35 & -2.15 & $0.03^{*}$ & $(-1.44,-0.06)$ \\
Block order & -1.21 & 0.92 & -1.32 & 0.18 & $(-3.02,0.58)$ \\
Sex $\times$ Cue & -3.61 & 1.85 & -1.94 & $0.05^{*}$ & $(-7.24,0.02)$ \\
Sex $\times$ Mobility & 0.05 & 0.07 & 0.79 & 0.42 & $(-0.08,0.20)$ \\
Sex $\times$ Block Order & 2.38 & 1.27 & 1.87 & 0.06 & $(-0.10,4.88)$ \\
Sex $\times$ Trial Number & -0.17 & 0.17 & -1.00 & 0.31 & $(-0.51,0.16)$ \\
Cue $\times$ Mobility & -0.10 & 0.04 & -2.41 & $0.01 *$ & $(-0.18,-0.01)$ \\
Sex $\times$ Cue $\times$ Mobility & 0.15 & 0.06 & 2.58 & $.009^{*}$ & $(0.03,0.28)$ \\
\hline
\end{tabular}




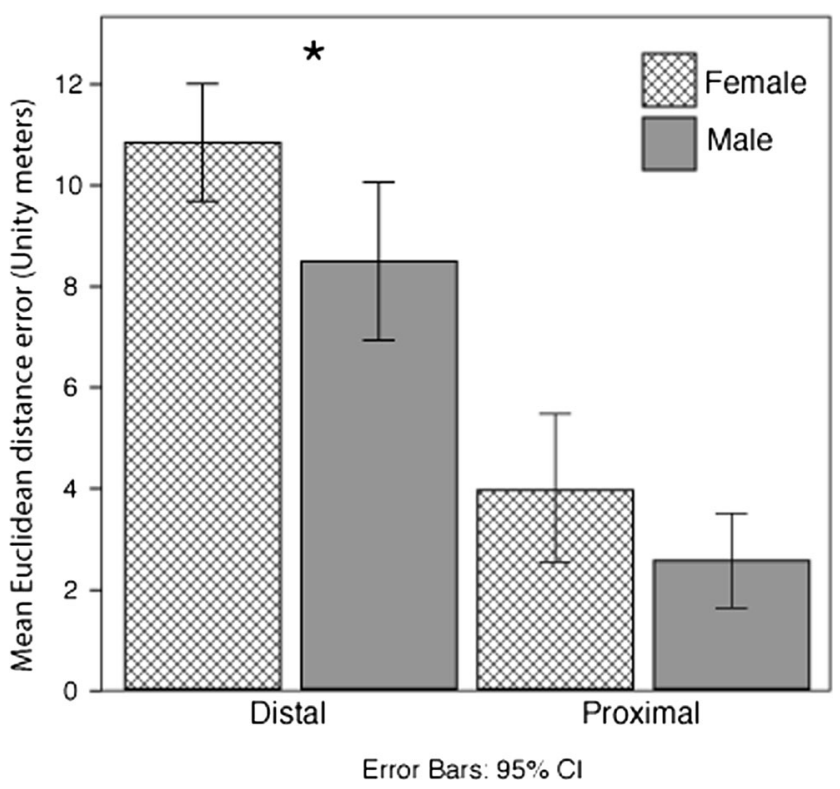

Fig. 3 Euclidean distance error as a function of distal or proximal cues and sex, for the small-scale maze. Error bars represent $95 \%$ confidence intervals. $*=p<.05$

to or use the distal cues at all (Livingstone-Lee et al., 2011) and thus the effects of mobility experience only appeared in our proximal cue condition.

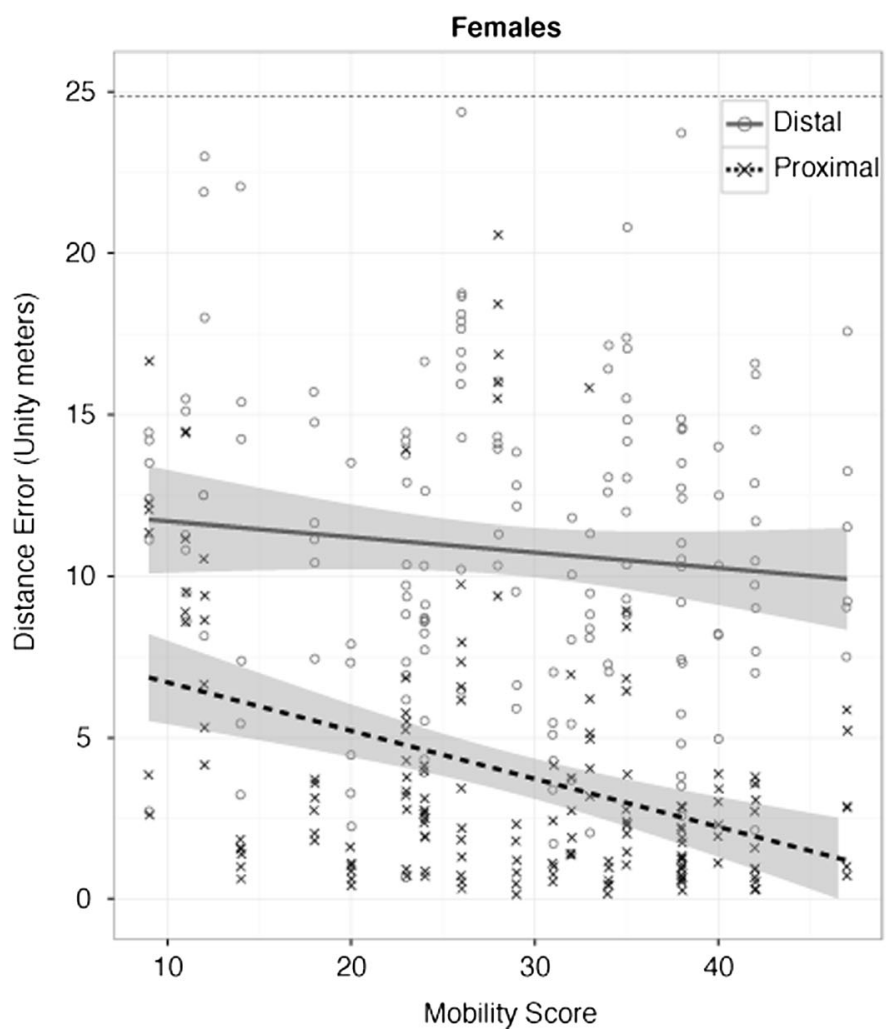

Fig. 4 Euclidean distance error as a function of mobility scores (as assessed by self-report of number of locations traveled) for the males and females in the distal and proximal cue conditions for the small-
The same mixed effects analysis was conducted for the large-scale maze (see Table 2). Our results showed that males $(M=18.87, S E=1.56)$ had significantly fewer navigation errors than females $(M=33.66, S E=1.72$; see Fig. 5). However, there was no interaction between sex and cue as found in the small-scale analysis, suggesting that the male advantage was consistent across both cue conditions. In addition, there was a significant Cue $\times$ Mobility interaction (see Fig. 6), revealing that Mobility reduced errors in the proximal condition more than the distal condition across both males and females. The difference in results of the two scales suggests that mobility experience affected navigation performance for both males and females in the larger scale, but specifically affected female performance on the smaller scale. Mobility may not have facilitated male performance on the smallscale maze because of the overall reduced error level for males, particularly for the proximal cue condition.

\section{General discussion}

In our small-scale maze, we replicated the work of Chai and Jacobs (2009), showing that males display superior performance compared to females when navigating with

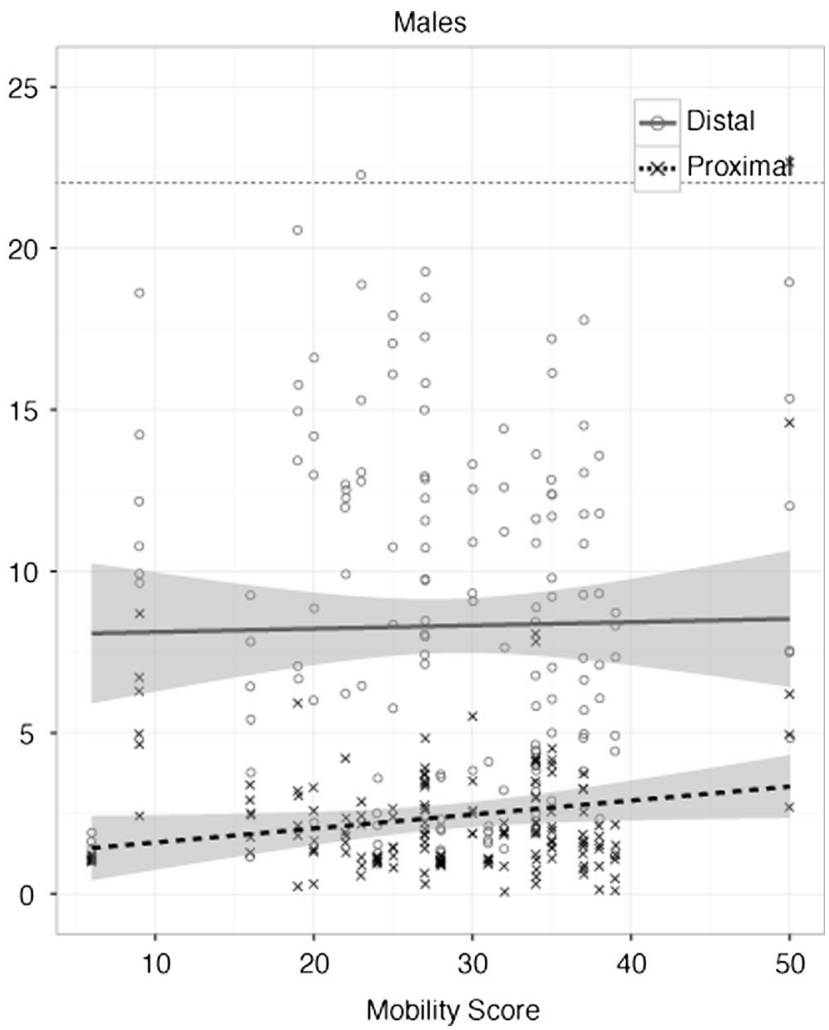

scale maze. The gray bands indicate $95 \%$ confidence intervals. The dotted line at the top of the figure represents 3 standard deviations above mean error 
Table 2 List of fixed effects with coefficients, standard errors, $p$ values, and $95 \%$ confidence intervals from the statistical model for the large-scale maze. Coefficients for interactions including Cue indicate the change from distal to proximal cues. Coefficients for interactions including Sex indicate the change from female to male. Coefficients for interactions including Block Order indicate the change from proximal then distal to distal then proximal

\begin{tabular}{lrlcll}
\hline Fixed effect & Estimate & Standard error & $t$-ratio & $p$-value & $95 \%$ CI \\
\hline Sex & -20.85 & 7.29 & -2.85 & $.004^{*}$ & $(-33.65,-6.69)$ \\
Cue & -13.71 & 3.88 & -3.53 & $.000^{*}$ & $(-21.32,-6.10)$ \\
Mobility & 0.04 & 0.13 & 0.33 & 0.73 & $(-0.22,0.32)$ \\
Trial number & -1.84 & 0.49 & -3.70 & $.000^{*}$ & $(-2.42,-1.06)$ \\
Gaming & -3.31 & 1.23 & -2.69 & $.007^{*}$ & $(-5.72,-0.90)$ \\
Block order & 4.13 & 3.00 & 1.37 & .168 & $(-1.75,10.02)$ \\
Sex $\times$ Cue & 2.25 & 6.24 & 0.36 & 0.71 & $(-9.99,14.50)$ \\
Sex $\times$ Mobility & 0.15 & 0.20 & 0.77 & 0.44 & $(-0.24,0.56)$ \\
Sex $\times$ Block Order & 0.67 & 4.21 & 0.15 & 0.87 & $(-7.58,8.92)$ \\
Sex $\times$ Trial Number & 0.19 & 0.69 & 0.27 & 0.78 & $(-0.64,-0.10)$ \\
Cue $\times$ Mobility & -0.37 & 0.13 & -2.69 & $.006 *$ & $(-0.64,-0.10)$ \\
Sex $\times$ Cue $\times$ Mobility & 0.29 & 0.20 & 1.49 & 0.13 & $(-0.09,0.69)$ \\
\hline
\end{tabular}

$*=<.05$ distal cues and that there was no sex difference when navigating with proximal cues. In the large-scale maze, we found that males displayed superior performance when navigating with both distal and proximal cues compared to females. These findings support the argument that spatial abilities used in small-scale spaces may not generalize fully to spatial abilities used in larger scale spaces (Hegarty et al., 2006; Learmonth et al., 2002; Montello, 1993; Wolbers \& Wiener, 2014). Much of the previous work on navigation and memory across different scales has distinguished between vista spaces-smaller, typically "room-size" spaces that can be fully seen from a single viewing location-and environmental

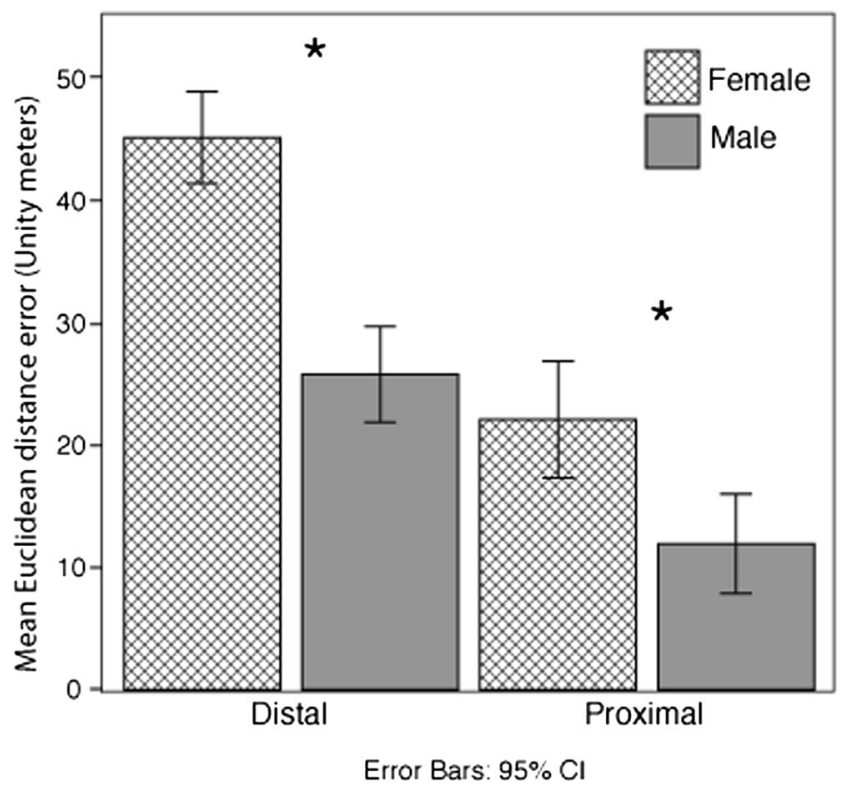

Fig. 5 Euclidean distance error as a function of sex, for the large-scale maze. Error bars represent $95 \%$ confidence intervals. $*=p<.05$ spaces, larger spaces where it is necessary to traverse the space in order to view all of the cues (Montello, 1993). In this sense, scale and visibility are often confounded-smaller spaces have cues visible from a single viewpoint and studies with larger spaces have been designed with paths or rooms that do not share visible cues (Meilinger et al., 2014). Our study is a beginning step to examine the influence of scale on navigation independent of visibility. The current results that are based on a very large open space where cues are at least visible (if not memorable), add to the growing literature on spatial abilities across different scales by showing that individual differences do not fully generalize from small to large scales, even when the visual cues are very similar.

Although the same cues were used in the small- and largescale mazes, the utility of the cues may have changed (see Fig. 7 for a screenshot of the proximal cues in the largescale maze). This could explain the sex difference in the

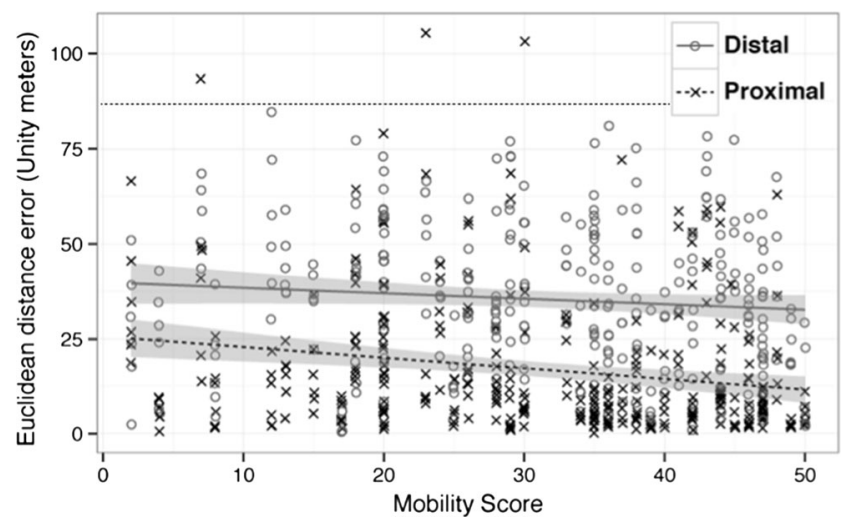

Fig. 6 Euclidean distance error as a function of mobility scores for the proximal and distal cue conditions, in the large-scale maze. The gray bands indicate $95 \%$ confidence intervals. The dotted line at the top of the figure represents 3 standard deviations above mean error 
Fig. 7 Screenshot of large-scale proximal condition, which shows eight cues

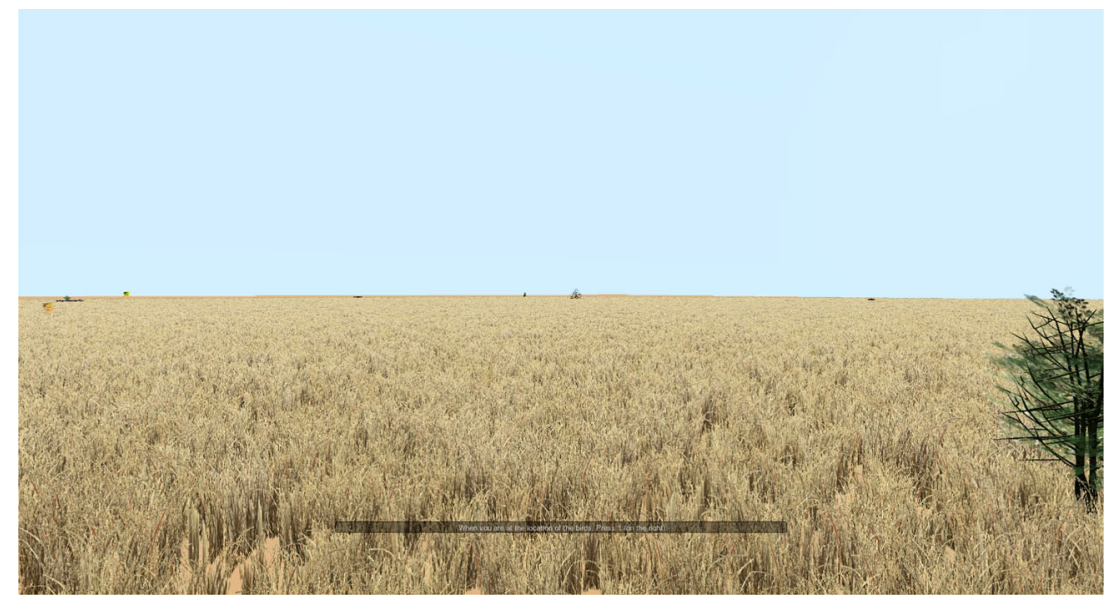

proximal-cue condition found for the large maze (but not the small maze or previous related work). To keep the relative distances, sizes, and quantity of the cues consistent across scale, we multiplied the distances between the cues in the small-scale maze by four. This increased the distances between the proximal cues by roughly $15 \mathrm{~m}$. The longer distance between cues may have resulted in females no longer being able to use their proximal cue strategies effectively in the large-scale maze. To our knowledge, there are no studies that identify distances that differentiate proximal and distal cues. In an extensive review, Knierim and Hamilton (2011) define proximal cues operationally as those inside the maze apparatus, whereas distal are those outside the apparatus. However, cues outside the apparatus can be both relatively close (e.g., Sandstrom et al., 1998), and far (e.g., Chai \& Jacobs, 2009). In addition to the distance from the viewer, the distinct effects of salience and visual angle for proximal and distal cues are unclear. Future work is needed to define the properties and context of distal and proximal cues that enable male and female navigation abilities.

We did find an influence of navigation experience on cue use in both the large- and small-scale mazes. Overall, we found that navigation experience related positively to accuracy on the maze and interacted with effects of sex, but there were differences between the two scales. In the small-scale maze, for females only, error decreased as mobility experience increased for the proximal cue condition. In the large maze, all participants showed improved performance with increased mobility experience, but again only in the proximal cue condition. While we had no predictions of how navigation experience might differentially affect the cue conditions, the results suggest that strategies that are developed through experience with travel to new places rely more on the information gained from proximal cues.

It is also possible that our measure of navigation experience was unable to identify important components of mobility that might influence navigation with distal cues. For example, being the navigator or the passenger, locomoting by different means (e.g., car, plane, bike, on foot), or using GPS devices could yield different types of navigation experience. Although additional work needs to be conducted to develop our measure of navigation experience and to understand which components of travel may influence spatial skills, the current findings are suggestive of a positive relationship between navigation experience and navigational skills that help to explain some of the disparities typically seen between women and men in large-scale navigation tasks.

Author Note This research is based upon work supported by the National Science Foundation under Grant No. 1329091.

\section{References}

Astur, R. S., Ortiz, M. L., \& Sutherland, R. J. (1998). A characterization of performance by men and women in a virtual Morris water task: A large and reliable sex difference. Behavioural Brain Research, 93(1-2), 185-190.

Bates, D., M. Maechler \& B. Bolker (2012). lme4: Linear mixed-effects models using S4 classes. Retrieved from http://CRAN.R-project. org $/$ package $=$ lme4 (R package version 0.999999-0)

Cashdan, E., \& Gaulin, S. J. (2016). Why go there? Evolution of mobility and spatial cognition in women and men. Human Nature, 27,(1), 115.

Chai, X. J., \& Jacobs, L. F. (2009). Sex differences in directional cue use in a virtual landscape. Behavioral Neuroscience, 123(2), 276-283.

Chai, X. J., \& Jacobs, L. F. (2010). Effects of cue types on sex differences in human spatial memory. Behavioural Brain Research, 208(2), 336-342.

Choi, J., McKillop, E., Ward, M., \& L’Hirondelle, N. (2006). Sex-specific relationships etween route-learning strategies and abilities in a largescale environment. Environment and Behavior, 38(6), 791-801.

D’Hooge, R., \& De Deyn, P. P. (2001). Applications of the Morris water maze in the study of learning and memory. Brain Research Reviews, 36(1), 60-90.

Ecuyer-Dab, I., \& Robert, M. (2004). Spatial ability and home-range size: Examining the relationship in Western men and women (Homo sapiens). Journal of Comparative Psychology (Washington, D.C.: 1983), 118(2), 217-231.

Hegarty, M., Montello, D. R., Richardson, A. E., Ishikawa, T., \& Lovelace, K. (2006). Spatial abilities at different scales: Individual 
differences in aptitude-test performance and spatial-layout learning. Intelligence, 34(2), 151-176.

Jones, C., Braithwaite, V., \& Healy, S. (2003). The evolution of sex differences in spatial ability. Behavioral Neuroscience, 117(3), 403-411.

Knierim, J., \& Hamilton, D. (2011). Framing spatial cognition: neural representations of proximal and distal frames of reference and their roles in navigation. Physiological Reviews, 91(4), 1245-1279.

Learmonth, A. E., Nadel, L., \& Newcombe, N. S. (2002). Children's use of landmarks: Implications for modularity theory. Psychological Science, 13(4), 337-341.

Learmonth, A. E., Newcombe, N. S., Sheridan, N., \& Jones, M. (2008). Why size counts: Children's spatial reorientation in large and small enclosures. Developmental Science, 11(3), 414-426.

Lawton, C. (1994). Gender differences in way-finding strategies: Relationship to spatial ability and spatial anxiety. Sex Roles, 30(11-12), 765-779.

Livingstone-Lee, S., Murchison, S., Zeman, P., Gandhi, M., van Gerven, D., Stewart, L., ... Skelton, R. (2011). Simple gaze analysis and special design of a virtual Morris water maze provides a new method for differentiating egocentric and allocentric navigational strategy choice. Behavioural Brain Research, 225(1), 117-125.

MacDonald, D., \& Hewlett, B. (1999). Reproductive Interests and Forager Mobility 1. Current Anthropology, 40(4), 501-524.

Meilinger, T., Riecke, B., \& Bülthoff, H. (2014). Local and global reference frames for environmental spaces. The Quarterly Journal of Experimental Psychology, 67(3), 542-569.

Montello DR (1993) Scale and multiple psychologies of space. In: Frank AU, Campari I (eds) Spatial Information Theory - A Theoretical
Basis for GIS, Springer-Verlag, Berlin, Heidelberg, New York, pp 312-321

Morris, R. (1984). Developments of a water-maze procedure for studying spatial learning in the rat. Journal of Neuroscience Methods, 11(1), $47-60$.

Nadel, L., \& Hardt, O. (2004). The spatial brain. Neuropsychology, 18(3), 473.

O'Keefe, J., \& Nadel, L. (1978). The hippocampus as a cognitive map (Vol. 3, pp. 483-484). Oxford: Clarendon Press.

Sandstrom, N., Kaufman, J., \& Huettel, S. A. (1998). Males and females use different distal cues in a virtual environment navigation task. Cognitive Brain Research, 6(4), 351-360.

Santos, A., McGuckin, N., Nakamoto, H. Y., Gray, D., \& Liss, S. (2011). Summary of travel trends: 2009 national household travel survey (No. FHWA-PL-11-022).

Tolman, E. (1948). Cognitive maps in rats and men. Psychological Review, 55(4), 189.

Unity Technologies (2015). Unity 5 Professional Edition. San Francisco, California. http://unity3d.com/get-unity

Vashro, L., \& Cashdan, E. (2015). Spatial cognition, mobility, and reproductive success in northwestern Namibia. Evolution and Human Behavior, 36(2), 123-129.

Vashro, L., Padilla, L., \& Cashdan, E. (2016). Sex differences in mobility and spatial cognition. Human Nature, 27(1), 16-34.

Vorhees, C., \& Williams, M. (2006). Morris water maze: Procedures for assessing spatial and related forms of learning and memory. Nature Protocols, 1(2), 848-858.

Wolbers, T., \& Wiener, J. (2014). Challenges for identifying the neural mechanisms that support spatial navigation: the impact of spatial scale. Frontiers in Human Neuroscience, 8, 571. 